\title{
Atividade de limpeza e clientes de Elacatinus figaro (Pisces: Gobiidae) nos recifes de coral dos Parrachos de Muriú, Nordeste do Brasil
}

\author{
Carlos Eduardo Costa Campos $^{1,2}$ \& Júlio César Sá-Oliveira \\ ${ }^{1}$ Laboratório de Zoologia, Departamento de Ciências Biológicas, Universidade Federal do Amapá- \\ UNIFAP, Rod. Juscelino Kubitschek, Km 02, Jardim Marco Zero, CEP 68902-280, Macapá, AP, Brasil \\ ${ }^{2}$ Autor para correspondência: Carlos Eduardo Costa Campos,e-mail: ceccampos@unifap.br
}

CAMPOS, C.E.C. \& SÁ-OLIVEIRA, J.C. Cleaning activity and fish clients of Elacatinus figaro (Pisces: Gobiidae) on coral reefs of Parrachos de Muriú, Northeastern Brazil. Biota Neotrop. 11(1): http://www. biotaneotropica.org.br/v11n1/en/abstract?article+bn01011012011.

\begin{abstract}
Cleaner fishes remove ectoparasites, injured tissue, mucus and scales from the body surface of other fishes. This behavior is important for the maintenance and health of reef fish species. In the Brazilian coast there are 24 cleaner fish species, with Elacatinus figaro (a goby endemic to Brazil) being recognized as one of the most specialized. This study records the diversity of clients and the daily cleaning activity of Elacatinus figaro on coral reefs of Parrachos de Muriú, Rio Grande do Norte, Northeast Brazil. A total of 21 species belonging to 11 families were recorded, with 15 species of clients (68.4\% of total) active during the day, five (25.3\%) with nocturnal activity and one $(5.3 \%)$ with diurnal and nocturnal activity. The most frequent clients recorded were grunts (Haemulidae; 25.6\% of all cleaning events) and damselfishes (Pomacentridae; 22.8\%), whereas planktivores/ invertivores was the most frequently attended trophic category (23.6\%). Cleaning activity started between $05 \mathrm{~h} 14-$ 06h28 AM and ended between 5h13-5h25 PM. Size of clients ranged 7-40 cm (total length) and most individuals were medium-sized $(12-30 \mathrm{~cm})$. A total of $127 \pm 3$ cleaning events and $34 \pm 1.7$ minutes of cleaning activity were estimated per cleaning station per day (2-6 individuals of E. figaro).
\end{abstract}

Keywords: cleaner goby, Gobiidae, symbiosis, brazilian reef fishes, cleaning behaviour.

CAMPOS, C.E.C. \& SÁ-OLIVEIRA, J.C. Atividade de limpeza e clientes de Elacatinus figaro (Pisces: Gobiidae) nos recifes de coral dos Parrachos de Muriú, Nordeste do Brasil. Biota Neotrop. 11(1): http:// www.biotaneotropica.org.br/v11n1/pt/abstract?article+bn01011012011.

Resumo: Peixes limpadores removem ectoparasitas, tecido doente ou ferido, muco e escamas da superfície corporal de outros peixes, sendo portanto de fundamental importância para a manutenção do equilíbrio e da saúde dos peixes do ecossistema recifal. Na costa brasileira são registradas 24 espécies de peixes limpadores, das quais se destaca Elacatinus figaro, uma das espécies de limpadores mais especializada. Este estudo registrou a diversidade de clientes e o período de atividade de limpeza de Elacatinus figaro nos recifes de coral dos Parrachos de Muriú, Rio Grande do Norte. Foram registradas 21 espécies de clientes pertencentes a 11 famílias, das quais 15 espécies $(68,4 \%$ do total de espécies) são ativas durante o dia, cinco (25,3\%) têm atividade noturna e uma (5,3\%) atividade diurna e noturna. Os clientes mais frequentes de E. figaro foram espécies de Haemulidae $(25,6 \%)$ e Pomacentridae $(22,8 \%)$ e a categoria trófica planctófago/invertívoro foi a mais representativa (23,6\%). A atividade de limpeza teve início entre $05 \mathrm{~h} 14$ e $06 \mathrm{~h} 28$ e término entre $17 \mathrm{~h} 13$ e $17 \mathrm{~h} 25$. Os tamanhos dos clientes de E. figaro variaram de 7-40 cm com média de 12-30 cm de comprimento total. Um total de $127 \pm 3$ interações de limpeza e $34 \pm 1,7$ minutos utilizados na limpeza por estação por dia (2-6 indivíduos de E. figaro) foi registrado Palavras-chave: góbio limpador, Gobiidae, simbiose, peixes recifais brasileiros, comportamento de limpeza. 


\section{Introdução}

A simbiose de limpeza é uma das associações alimentares mais importantes, evidentes e complexas em comunidades de peixes recifais (Hobson 1971, Losey 1972, 1978), por envolver uma grande diversidade de espécies e eventos comportamentais muito conspícuos (Feder 1966, Losey 1971). A simbiose de limpeza tem sido considerada como mutualismo quando a incidência de ectoparasitas nos clientes é alta, e como comensalismo, ou mesmo parasitismo, quando a incidência é baixa (Poulin 1993, Grutter \& Poulin 1998).

Os peixes limpadores apresentam o hábito de remover ectoparasitas (fungos, bactérias e crustáceos), tecido doente, muco e escamas provenientes do corpo de outros peixes e invertebrados (Johnson 1982, Losey 1987, DeLoach 1999) em locais denominados estações de limpeza, onde os clientes frequentemente adotam uma posição característica enquanto os limpadores realizam sua atividade (DeLoach 1999). Este comportamento é considerado de fundamental importância para a manutenção do equilíbrio e da saúde dos peixes no ecossistema recifal (DeLoach 1999).

As espécies de peixes limpadores podem pertencer a duas categorias funcionais: limpador obrigatório, que realiza esta atividade durante toda a sua vida, e limpador facultativo, que limpa apenas quando juvenis (Côté 2000). O grau de especialização dos limpadores obrigatórios varia espacial e temporalmente numa mesma espécie (Whiteman \& Côté 2002), o que promove um maior ou menor grau de especialização. A flexibilidade deste comportamento depende ainda de diversas circunstâncias ecológicas e das oportunidades alimentares (Arnal \& Côté 1998, 2000).

O hábito alimentar limpador é descrito em cerca de 100 espécies de peixes, distribuídas em 19 famílias (Côté 2000). Dentre estas, destaca-se a família Gobiidae, que apresenta algumas espécies limpadoras, incluindo espécies do gênero Elacatinus. No Brasil, Elacatinus figaro é conhecida como uma das espécies de peixe limpador mais especializada e que tem como clientes mais frequentes espécies de Pomacentridae e Haemulidae (Sazima et al. 2000).

No Brasil existem diversos estudos sobre animais limpadores, como peixes (e.g. Sazima et al. 1997, 1998, 1999a, 1999b, 2000, Sazima \& Moura 2000, Francini-Filho et al. 2000, Feitoza et al. 2002, Guimarães et al. 2004, Campos et al. 2007, Francini-Filho \& Sazima 2008) e camarões (Sazima et al. 2004). Desta forma, foram registradas a diversidade, o período de atividade de limpeza e as categorias tróficas de clientes de Elacatinus figaro nos recifes de coral dos Parrachos de Muriú, Rio Grande do Norte, gerando dados que servem como base comparativa para um estudo similar realizado em costões rochosos do sudeste do Brasil (Sazima et al. 2000).

\section{Material e Métodos}

\section{1. Área de estudo}

Os recifes da costa do Rio Grande do Norte formam uma bancada de recifes costeiros denominados Parrachos de Muriú. Eles ocorrem sob a forma de corpos calcários lineares, descontínuos, dispostos em três linhas sucessivas paralelas entre si e a atual linha de costa, sendo a primeira linha, quase sempre emersa, próxima à praia e as demais parcialmente emersas (Leão et al. 2003).

Os Parrachos de Muriú abrangem uma área de $6 \mathrm{~km}$ de comprimento por $1 \mathrm{~km}$ de largura, distando menos de um $1 \mathrm{~km}$ da praia, com profundidade de 1 a $4 \mathrm{~m}$ da linha d' água ao fundo arenoso na baixa-mar. A base dos Parrachos de Muriú é arenítica, sobre a qual estão incrustados corais, algas calcárias e vermetídeos, sendo os corais Siderastrea stellata e Montastrea cavernosa os principais construtores (Maida \& Ferreira 1997). Sua superfície é recoberta também pelo zoantídeo Palythoa caribaeorum e macroalgas no fundo e em volta dos recifes.
São reconhecidas nos Parrachos de Muriú cinco feições morfológicas características: i) planície arenosa, ii) crista recifal traseira, iii) crista recifal frontal, iv) cristas e ranhuras e v) recifes em mancha, comuns às várias áreas estudadas e sua respectiva ictiofauna (Figura 1).

\section{Coleta de dados}

As sessões de observações foram realizadas durante o período de janeiro a dezembro de 2007 através de mergulho livre para áreas com profundidade média de até $4 \mathrm{~m}$, e mergulho autônomo para áreas além dos $5 \mathrm{~m}$ de profundidade. Foram realizadas 48 sessões diurnas com duração de 3 horas, totalizando 700 horas de observação e 24 observações crepusculares com duração de 2 horas, totalizando 300 horas. Durante as sessões foram registradas as espécies e o tamanho dos clientes envolvidos na atividade de limpeza. Posteriormente os clientes foram classificados de acordo com sua categoria trófica e o período de atividade (espécies diurnas, noturnas e diurnas/noturnas) (cf. Randall 1967, Sazima et al. 2000, Sazima \& Sazima 2001). Nas sessões de observações foram utilizados os métodos de "animal focal" e "todas as ocorrências" (cf. Altmann 1974, Diamant \& Spighel 1985).

A frequência e o tempo total médio da atividade de limpeza foram calculados através das médias das interações por minuto durante as horas do dia. Esta informação foi usada para estimar o tempo total utilizado pelos limpadores em atividades da limpeza por estação da limpeza durante o dia. As interações de limpeza foram consideradas como o período da associação entre E. figaro e os clientes, desde o começo do contato físico até a retirada do cliente ou do limpador (Johnson \& Ruben 1988). O Comprimento Total (CT) de cada cliente foi estimado através de uma régua de $50 \mathrm{~cm}$ de comprimento graduada em $1 \mathrm{~cm}$ e presa a um bastão ou através de objetos de tamanho conhecido (e.g. rocha ou parte de alga) próximos da estação de limpeza o do cliente. Algumas vezes o tamanho do cliente foi estimado usando o tamanho do limpador que estava interagindo com o cliente.

Todas as espécies de peixes recifais próximas à estação de limpeza durante o período de estudo foram registradas visando a obtenção de uma lista de clientes potenciais no local de estudo, sendo considerados clientes potenciais os peixes com hábitos gerais similares e registrados como um cliente de qualquer espécie de limpador do Atlântico Sul Ocidental (Sazima et al. 1997, 1998, Sazima \& Moura 2000, FranciniFilho \& Sazima 2008).

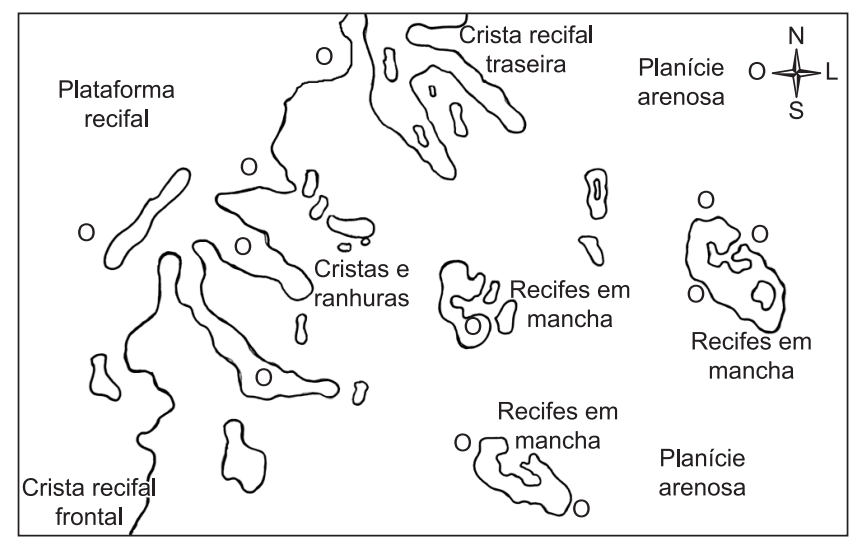

Figura 1. Parrachos de Muriú, Rio Grande do Norte, com as principais feições morfológicas amostradas. Os círculos indicam os locais das estações de limpeza de E. figaro. Recifes em mancha e plataforma recifal.

Figure 1. Parrachos de Muriú Reef, Rio Grande do Norte, with indication to the main morphological features sampled. The circles show the locations of cleaning stations of E. figaro. Patch reefs and reef flat. 


\section{Resultados}

Das cinco feições morfológicas características dos Parrachos de Muriú, Elacatinus figaro foi observado em apenas duas feições localizadas na margem Norte dos Parrachos: recifes em mancha e plataforma recifal. Cada estação de limpeza foi constituída por dois a seis limpadores. Foram registradas 21 espécies de clientes pertencentes a 11 famílias, das quais 15 espécies são ativas durante o dia $(68,4 \%)$, cinco têm atividade noturna $(25,3 \%)$ e uma atividade diurna e noturna $(5,3 \%)$. Com relação à categoria trófica, quatro espécies de clientes são carnívoras/invertívoras (19,1\%), duas planctófagas/invertívoras $(9,5 \%)$, cinco invertívoras $(23,8 \%)$, cinco onívoras $(23,8 \%)$, quatro herbívoras $(19,1 \%)$ e duas planctófagas $(4,8 \%)$ (Figura 2 e 3 ).

Foram registradas 27 espécies de clientes potenciais de $E$. figaro nos Parrachos de Muriú, das quais 21 foram observadas em associação de limpeza (Tabela 1). Os clientes de E. figaro mais frequentes foram espécies de Haemulidae (25,6\%) e Pomacentridae $(22,8)$ e de categoria trófica planctófago/invertívoro $(23,6 \%)$. As espécies atendidas mais frequentemente por E. figaro foram Haemulon aurolineatum (16,8\%), Abudefduf saxatilis $(15,6 \%)$ e Pseudupeneus maculatus $(8,8 \%)$, respectivamente.

A frequência de interações de limpeza variou durante todo o dia. As frequências mais elevadas foram observadas pela manhã (0506 horas) com 0,78 interações por minuto e à tarde (16-17 horas) com 0,47 interações, ao passo que as frequências mais baixas foram registradas ao meio-dia (0,13 interação por o minuto) (Figura 4).

A atividade de limpeza ocorreu durante todo o dia, tendo início entre $05 \mathrm{~h} 14$ e $06 \mathrm{~h} 28$ horas e término entre $17 \mathrm{~h} 13$ e $17 \mathrm{~h} 25$ horas. Os menores clientes de E. figaro (Média, desvio padrão e número de indivíduos) foram Canthigaster figueiredoi (7,9 \pm 0,7 $\mathrm{n}=6)$ e Chaetodon striatus $(10,1 \pm 1,2 \mathrm{n}=12)$ e os maiores foram Scarus zelindae $(35,1 \pm 3,2 \mathrm{n}=18)$, Halichoeres brasiliensis $(28,8$ $\pm 1,1 \mathrm{n}=15)$ e Bodianus rufus $(27,2 \pm 6,9 \mathrm{n}=8)$.

\section{Discussão}

O número de clientes de E. figaro registrados por Sazima et al. (2000) em recifes do Sudeste do Brasil (34 espécies), por FranciniFilho \& Sazima (2008) para E. cf. randalli (22 espécies) e por

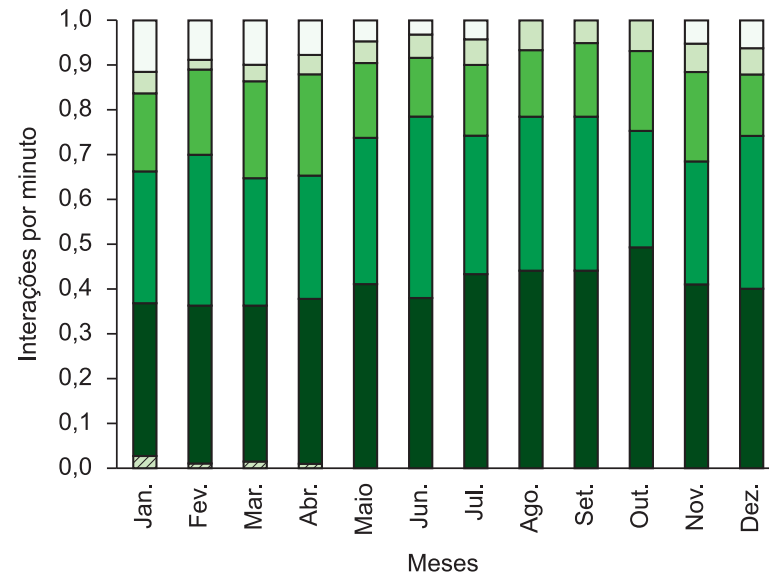

$\begin{array}{lll}\square \text { Herbívoro } & \square \text { Onívoro } & \square \text { Invertívoro } \\ \square \text { Planctófago } & \square \text { Planctófago/Invertívoro } & \square \text { Carnívoro/Invertívoro }\end{array}$

Figura 2. Frequência de eventos de limpeza de E. figaro nos Parrachos de Muriú, Rio Grande do Norte. Interações de limpeza ( $\mathrm{min}$ ) por categoria trófica.

Figure 2. Frequency of cleaning events of E. figaro on coral reefs of Parrachos de Muriú, Rio Grande do Norte. Cleaning interactions (min) by trophic category.

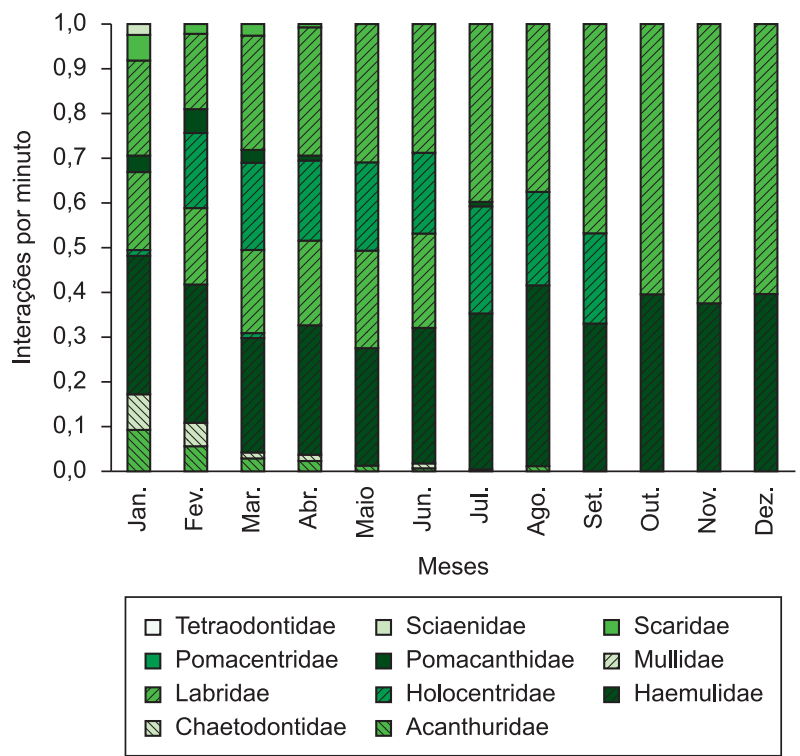

Figura 3. Frequência de eventos de limpeza de E. figaro nos Parrachos de Muriú, Rio Grande do Norte. Interações de limpeza (minuto) por categoria taxonômica.

Figure 3. Frequency of cleaning events of E. fígaro on coral reefs of Parrachos de Muriú, Rio Grande do Norte. Cleaning interactions (minute) by taxonomic category.
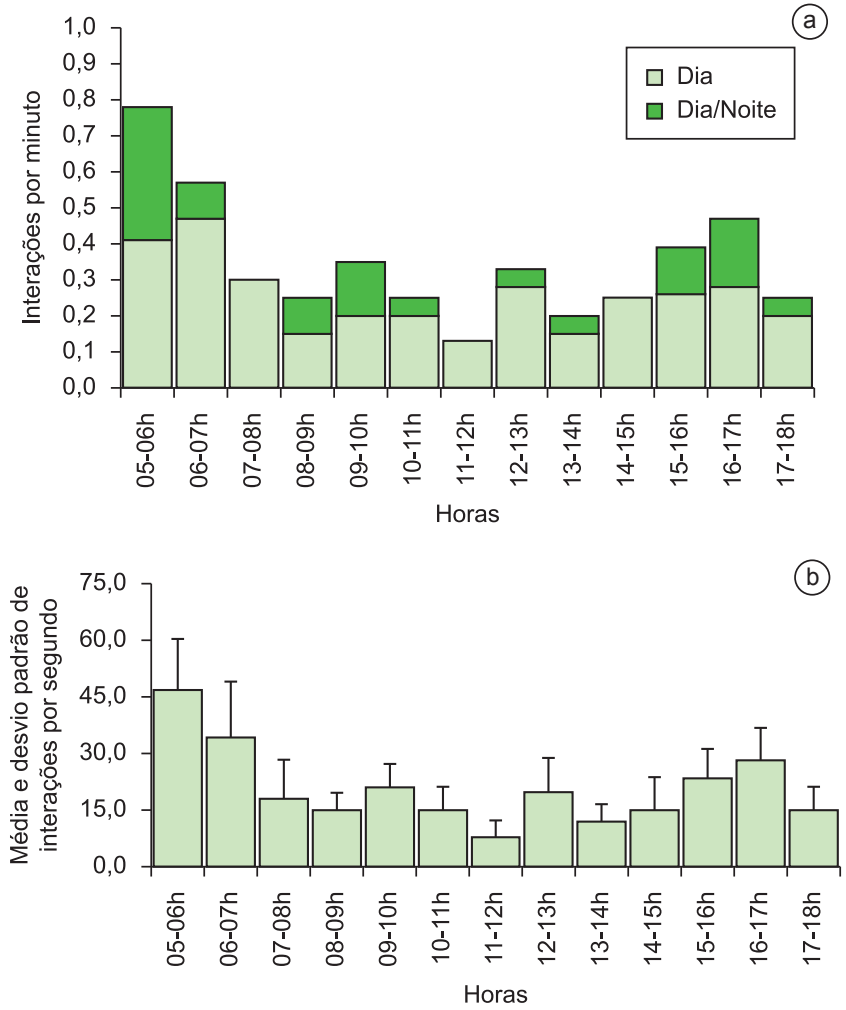

Figura 4. Frequência de eventos de limpeza de E. figaro nos Parrachos de Muriú, Rio Grande do Norte, mostrando a frequência de a) interações por minuto de espécies diurnas e noturnas; e b) média e desvio padrão de interações por segundo.

Figure 4. Frequency of cleaning events of E. fígaro on coral reefs of Parrachos de Muriú, Rio Grande do Norte, showing the frequency of a) interactions by minutes of diurnal and nocturnal species; and b) the mean \pm 1 SD interactions by seconds. 
Campos, C.E.C. \& Sá-Oliveira, J.C.

Tabela 1. Clientes de E. figaro registrados em 250 eventos de limpeza nos Parrachos de Muriú, Rio Grande do Norte. Famílias, período de atividade, espécies, categoria trófica, tamanho do peixe (cm; TL) e frequência de eventos de limpeza (\% do total de eventos). \# clientes potenciais de E. figaro registrados nas estações de limpeza.

Table 1. Reef fish species cleaned by the barber goby, Elacatinus figaro recorded over 250 cleaning events on coral reefs of Parrachos de Muriú, Rio Grande do Norte. Families, activity period, trophic category, size (total lenght) and frequency of cleaning events (\% of the total). \# potential clients of $E$. figaro recorded at the cleaning stations.

\begin{tabular}{|c|c|c|c|c|c|}
\hline Família & Período de atividade & Espécie & Categoria trófica & Tamanho $(\mathrm{cm})$ & Eventos de limpeza \\
\hline Holocentridae & Noturno & Holocentrus ascensionis & Carnívoro/Invertívoro & $20-32$ & 7 \\
\hline \multirow[t]{4}{*}{ Haemulidae } & Noturno & Anisotremus surinamensis & Carnívoro/Invertívoro & $20-28$ & 3 \\
\hline & Noturno & Anisotremus virginicus & Carnívoro/Invertívoro & $18-30$ & 2 \\
\hline & Diurno/noturno & Haemulon aurolineatum & Planctófago/Invertívoro & $10-18$ & 42 \\
\hline & Noturno & Haemulon plumieri & Invertívoro & $18-20$ & 17 \\
\hline Sciaenidae & Noturno & Odontoscion dentex & Carnívoro/Invertívoro & $12-15$ & 1 \\
\hline Mullidae & Diurno & Pseudupeneus maculatus & Planctófago/Invertívoro & $10-28$ & 22 \\
\hline Chaetodontidae & Diurno & Chaetodon striatus & Invertívoro & $8-12$ & 15 \\
\hline \multirow[t]{2}{*}{ Pomacanthidae } & Diurno & Holacanthus tricolor & Onívoro & 15 & 5 \\
\hline & Diurno & Pomacanthus paru & Onívoro & $28-30$ & 4 \\
\hline \multirow[t]{3}{*}{ Pomacentridae } & Diurno & Abudefduf saxatilis & Planctófago/Onívoro & $15-20$ & 39 \\
\hline & Diurno & Chromis multilineata & Planctófago & $10-14$ & 17 \\
\hline & Diurno & Stegastes fuscus & Onívoro & $12-14$ & 1 \\
\hline \multirow[t]{3}{*}{ Labridae } & Diurno & Bodianus rufus & Invertívoro & $18-40$ & 9 \\
\hline & Diurno & Halichoeres brasiliensis & Invertívoro & $28-32$ & 12 \\
\hline & Diurno & Halichoeres poeyi & Invertívoro & $12-20$ & 12 \\
\hline \multirow[t]{2}{*}{ Scaridae } & Diurno & Scarus zelindae & Herbívoro & $30-40$ & 6 \\
\hline & Diurno & Sparisoma aff. atomarium & Herbívoro & $20-28$ & 7 \\
\hline \multirow[t]{2}{*}{ Acanthuridae } & Diurno & Acanthurus chirurgus & Herbívoro & $22-28$ & 9 \\
\hline & Diurno & Acanthurus coeruleus & Herbívoro & 30 & 16 \\
\hline Tetraodontidae & Diurno & Canthigaster aff. rostrata & Onívoro & $7-9$ & 4 \\
\hline \multirow[t]{3}{*}{ Epinephilidae } & Diurno/Noturno & Epinephelus adscensionis & Carnívoro/Invertívoro & $10-25$ & \# \\
\hline & Diurno/Noturno & Cephalopholis fulva & Carnívoro/Invertívoro & $10-20$ & \# \\
\hline & Diurno/Noturno & Mycteroperca bonaci & Carnívoro/Invertívoro & $17-30$ & $\#$ \\
\hline \multirow[t]{2}{*}{ Lutjanidae } & Diurno/Noturno & Lujanus jocu & Carnívoro/Invertívoro & $15-30$ & $\#$ \\
\hline & Diurno & Lutjanus chrysurus & Carnívoro/Invertívoro & $15-25$ & \# \\
\hline Pempheridae & Noturno & Pempheris schomburgki & Planctófago & $8-15$ & $\#$ \\
\hline
\end{tabular}

Johnson \& Ruben (1988) para E. evelynae (24 espécies) excede o número de espécies registradas no presente estudo (21 espécies). O número reduzido de clientes registrados no presente estudo se deve possivelmente à pressão antrópica observada na área de estudo, que sofre influência da pesca ornamental artesanal e também é utilizada para fins recreativos devido a sua facilidade de acesso e proximidade da praia (Ferreira \& Cava 2001, Feitosa et al. 2002, Campos et al. 2010).

A baixa riqueza de espécies (123 espécies e 45 famílias) registrada por Campos et al. (2010) nos Parrachos de Muriú pode ter ocasionado a redução do número de clientes nas estações de limpeza de $E$. figaro e, consequentemente, limitado a sua atividade de limpeza, como constatado para outras espécies de limpadores (Wicksten 1995, Arnal \& Côté 1998).

O número de interações de limpeza por estação de E. figaro foram baixos quando comparados aos valores encontrados por Grutter (1997) e Johnson \& Ruben (1988). Para o limpador Labroides dimidiatus a variação diária de frequência de limpeza foi de $2297 \pm 83$ interações e $256 \pm 11$ minutos por dia gastos nessa atividade (Grutter 1997). Em uma estação de limpeza constituída pelas espécies Elacatinus evelynae, Bodianus rufus e Thalassoma bifasciatum aproximadamente 1.500 interações de limpeza foram registradas por dia (Johnson \& Ruben 1988). Em estudo realizado com a espécie E. figaro nas ilhas Anchieta e Vitória e na Laje de Santos (23 $33^{\text {' }}$ $-24^{\circ} 19^{\prime}$ S), Sazima et al. (2000) registraram um total de $109 \pm 3$ interações de limpeza e $30 \pm 1$ minutos utilizados na limpeza por estação por dia. No presente estudo foram observadas $127 \pm 3$ interações de limpeza e $34 \pm 1,7$ minutos utilizados na limpeza por estação por dia (2-6 indivíduos de E. figaro).

O estabelecimento de estações de limpeza favorece a localização dos limpadores pelos clientes, caracterizando os limpadores mais especializados (Brockmann \& Hailman 1976, Sazima et al. 1997). O período de atividade dos clientes está correlacionado com o ciclo diário de iluminação (Helfmann 1993), sendo considerada como uma interação estritamente diurna, devido aos clientes e os limpadores serem dependentes de sinais visuais (Losey 1971, 1978, 1987). Durante o período diurno a maior parte das espécies de peixes da comunidade recifal se encontra ativa, sendo composta por espécies conspícuas, de coloração viva e com grande número de indivíduos (Helfmann 1993). Haemulon aurolineatum foi o cliente mais frequente no período diurno e crepuscular, períodos que coincidem com a chegada de grupos para predar organismos planctônicos na coluna d'água (dia) e com a partida para vasculhar o substrato arenoso (crepúsculo). 
Estudos de atividade de limpeza no Caribe registraram a diversidade e a frequência de clientes atendidos pelo gênero Elacatinus em diversos locais durante o dia (e.g. Clepticus parra em St. Croix e Chromis multilineata em Barbados) (Johnson \& Ruben 1988, Wicksten 1995, Arnal \& Côté 1998), fato observado no presente estudo. O hábito dos planctófagos de se aglomerar nas estações de limpeza em grupos e sua elevada frequência estão relacionados provavelmente a sua incidência elevada como clientes durante o período diurno (Johnson \& Ruben 1988, Francini-Filho et al. 2000, Sazima et al. 2000). Espécies predominantemente planctófagas, indicam uma provável relação entre as categorias tróficas e a frequência dos eventos de limpeza (Arnal \& Côté 1998).

Embora os clientes dos peixes limpadores sejam compostos por peixes recifais diurnos (Sazima et al. 1999a, 2000) algumas espécies com hábitos noturnos também procuram os peixes limpadores (e.g. Pempheris schomburgki), ficando expostas a potenciais predadores diurnos quando precisam deixar seus abrigos (Sazima et al. 2005). Este estudo revela que apesar da diferença na localização geográfica, os padrões encontrados para E. figaro no sudeste e nordeste do Brasil são semelhantes.

\section{Referências Bibliográficas}

ALTMANN, J. 1974. Observational study of behavior: sampling methods. Behavior 49 (3-4):227-265.

ARNAL, C. \& CÔTÉ, I.M. 1998. Interactions between cleaning gobies and territorial damselfish on coral reefs. Anim. Behav. 55:1429-1442.

ARNAL, C. \& CÔTÉ, I.M. 2000. Diet of broadstripe cleaning gobies on a Barbadian reef. J. Fish Biol. 57:1075-1082.

BROCKMANN, H.J. \& HAILMAN, J.P. 1976. Fish cleaning symbiosis: notes on juvenile angelfishes (Pomacanthus, Chaetodontidae) and comparison with other species. Z. Tierpsychol. 42:129-138.

CAMPOS, C.E.C., SILVA, M.B., TARGINO, S.G., SÁ-OLIVEIRA, J.C. \& ARAÚJO, A.S. 2007. Simbiose de Limpeza de Thalassoma noronhanum (Boulenger, 1890) (Labridae) na Reserva Biológica do Atol das Rocas, Rio Grande do Norte, Brasil. Rev. Etol. 8(2):63-70.

CAMPOS, C.E.C., SÁ-OLIVEIRA, J.C. \& ARAÚJO, A.S. 2010. Composição e estrutura de comunidades de peixes nos Parrachos de Muriú, Estado do Rio Grande do Norte, Brasil. Arq. Cien. Mar 43(1):63-75.

CÔTÉ, I.M. 2000. Evolution and ecology of cleaning symbioses in the sea. Oceanogr. Mar. Biol. Annu. Rev. 38:311-355.

DELOACH, N. 1999. Reef fish behavior: Florida, Caribbean, Bahamas. New World Publications, Verona, Italy.

DIAMANT, A. \& SHPIGEL, M. 1985. Interspecific feeding associations of groupers (Teleostei: Serranidae) with octopuses and moray eels in the Gulf of Eilat (Aqaba). Environ. Biol. Fishes 13(2):153-159.

FEDER, H.M. 1966. Cleaning symbiosis in the marine environment. In Symbiosis (S.M. Henry, ed.). Academic Press, New York, p. 327-380.

FEITOSA, C.V., PIMENTA, D.A.S. \& ARAÚJO, M.E. 2002. Ictiofauna recifal dos Parrachos de Maracajaú (RN) na área dos flutuantes: inventário e estrutura de comunidades. Arq. Cien. Mar 35:133-138.

FEITOZA, B.M., DIAS, T.L.P., ROCHA, L.A. \& GASPARINI, J.L. 2002 First record of cleaning activity in the slippery dick, Halichoeres bivittatus (Perciformes: Labridae), off northeastern Brazil. J. Ichthyol. Aquat. Biol. 5:73-76.

FERREIRA, B.P. \& CAVA, F. 2001. Ictiofauna marinha da APA Costa dos Corais: lista de espécies através de levantamento da pesca e observações subaquáticas. Bol. Tec. Cient. CEPENE 9(1):167-180.

FRANCINI-FILHO, R.B., MOURA, R.L., \& SAZIMA, I. 2000. Cleaning by the wrasse Thalassoma noronhanum, with a record of predation by its grouper client Cephalopholis fulva. J. Fish Biol. 56(4):802-809.

FRANCINI-FILHO, R.B. \& SAZIMA, I. 2008. A comparative study of cleaning activity of two reef fishes at Fernando de Noronha Archipelago, tropical West Atlantic. Environ. Biol. Fishes 83:213-220.

GRUTTER, A.S. 1997. Spatiotemporal variation and feeding selectivity in the diet of the cleaner fish Labroides dimidiatus. Copeia 7:346-355.
GRUTTER, A.S. \& POULIN, R. 1998. Cleaning on coral reefs by the wrasse Labroides dimidiatus: influence of client body size and phylogeny. Copeia 1:120-127.

GUIMARÃES, R.Z.P., GASPARINI, J.L. \& ROCHA, L.A. 2004. A new cleaner goby of the genus Elacatinus (Teleostei: Gobiidae), from Trindade Island, off Brazil. Zootaxa 770:1-8.

HELFMAN, G.S. 1993. Fish behavior by day, night and twilight. In Behavior of teleost fishes (T.J. Pitche, ed.). Chapman \& Hall, London, p.479-512.

HOBSON, E.S. 1971. Cleaning symbiosis among California inshore fishes. Fish. Bull. 69:491-523.

JOHNSON, W.S. 1982. A record of cleaning symbiosis involving Gobiosoma sp. and a large Caribbean octopus. Copeia 3:712-714.

JOHNSON, W.S. \& RUBEN, P. 1988. Cleaning behavior of Bodianus rufus, Thalassoma bifasciatum, Gobiosoma evelynae and Periclimenes pedersoni along a depth gradient at Salt River Submarine Canyon, St. Croix. Environ. Biol. Fishes 23:225-232.

LEÃO, Z.M.A.N., KIKUCHI, R.K.P. \& TESTA, V. 2003. Corals and coral reefs of Brazil. In Latin American Coral reefs (J. Cortés, ed.). Chapman \& Hall, Amsterdam, p. 9-52.

LOSEY, G.S.J. 1971. Communication between fishes in cleaning symbiosis. In Aspects of biology of symbiosis (T.C. Cheng, ed.). University Park Press, Baltimore, p. 45-76.

LOSEY, G.S.J. 1972. The ecological importance of cleaning symbiosis. Copeia 4:820-833.

LOSEY, G.S.J. 1978. The symbiotic behavior of fishes. In The behavior of fish and other aquatic animals (T.C. Cheng, ed.). Academic Press, New York, p. 1-31.

LOSEY, G.S.J. 1987. Cleaning symbiosis. Symbiosis 4:229-258.

MAIDA, M. \& FERREIRA, B.P. 1997. Coral reefs of Brazil: an overview. In 8th International Coral Reef Symposium. 1: 263-274.

POULIN, R. 1993. A cleaner perspective on cleaning symbiosis. Rev. Fish Biol. Fish. 3:75-79.

RANDALL, J.E. 1967. Food habits of reef fishes of the West Indies. Stud. Trop. Oceanogr. 5:665-847.

SAZIMA, C. \& SAZIMA, I. 2001. Plankton-feeding aggregation and occasional cleaning by adult butterflyfish, Chaetodon striatus (Chaetodontidae) in Southwestern Atlantic. Cybium 25(2):145-151.

SAZIMA, C., BONALDO, R.M., KRAJEWSKI, J.P. \& SAZIMA, I. 2005. The Noronha wrasse: a "jack-of-all-trades" follower. J. Ichthyol. Aquat. Biol. 9:97-108

SAZIMA, I., MOURA, R.L. \& ROSA, R.S. 1997. Elacatinus figaro sp. n. (Perciformes: Gobiidae), a new cleaner goby from the coast of Brazil. J. Ichthyol. Aquat. Biol. 2:33-38

SAZIMA, I., MOURA, R.L. \& GASPARINI, J.L. 1998. The wrasse Halichoeres cyanocephalus (Labridae) as a specialized cleaner fish. Bull. Mar. Sci. 63:605-610.

SAZIMA, I., MOURA, R.L. \& SAZIMA, C. 1999a. Cleaning activity of juvenile angelfish, Pomacanthus paru, on the reefs of the Abrolhos Archipelago, western South Atlantic. Environ. Biol. Fishes 56:399-407.

SAZIMA, I., MOURA, R.L. \& RODRIGUES, M.C.M. 1999b. A juvenile suckerfish, Echeneis naucrates (Echeneidae), acting as a station-based cleaner fish. Cybium 23:377-380.

SAZIMA, I., SAZIMA, C., FRANCINI-FILHO, R.B. \& MOURA, R.L. 2000. Daily cleaning activity and diversity of clients of the barber goby, Elacatinus figaro, on rocky reefs in southeastern Brazil. Environ. Biol. Fishes 59:69-77.

SAZIMA, I. \& MOURA, R.L. 2000. Shark, Carcharhinus perezi, cleaned by the goby, Elacatinus randalli, at Fernando de Noronha Archipelago, western South Atlantic. Copeia 2000:297-299.

SAZIMA, I., GROSSMAN, A. \& SAZIMA, C. 2004. Tartarugas-de-pente visitam barbeiros bigududos: simbiose de limpeza entre Eretmochelys imbricata entre e o camarão Stenopus hispidus. Biota Neotrop. 4(1):1-6.

WICKSTEN, M.K. 1995. Associations of fishes and their cleaners on coral reefs of Bonaire, Netherlands Antilles. Copeia 9:477-481.

WHITEMAN, E.A. \& CÔTÉ, I.M. 2002. Cleaning activity of two Caribbean cleaning gobies: intra - and interspecific comparisons. J. Fish Biol. 60(6):14-43. 